\title{
Bacterial profile and drug resistance patterns in neonates admitted with sepsis to a tertiary teaching hospital in Ethiopia
}

\author{
Mulatu Gashaw ( $\nabla$ mulatugashaw@gmail.com ) \\ Jimma University https://orcid.org/0000-0002-2299-7863
}

\section{Solomon Ali}

School of Medical Laboratory Sciences, Jimma University and St Paul's Millennium Medical College, Addis Ababa,

Ethiopia

\section{Getnet Tesfaw}

School of Medical Laboratory Sciences, Jimma University

\section{Beza Eshetu}

Department of Pediatrics and Child Health, Jimma University, Ethiopia

\section{Netsanet Workneh}

Department of Pediatrics and Child Health, Jimma University, Ethiopia

\section{Melkamu Berhane}

Department of Pediatrics and Child Health, Jimma University, Ethiopia

\section{Guro K. Bårnes}

Division for Infection Control and Environmental Health, Norwegian Institute of Public Health, Oslo, Norway and Innlandet Hospital Trust, Division Gjøvik-Lillehammer, Gjøvik, Norway

\section{Guenter Froeschl}

Division of Infectious Disease and Tropical Medicine, University Hospital (LMU), Munich

\section{Andreas Wieser}

Max von Pattenkofer-Institute (Medical Microbiology), LMU

\section{Esayas Kebede Gudina}

Department of Internal Medicine, Jimma University, Ethiopia

\section{Research article}

Keywords: Sepsis, Neonate, Multidrug resistance

Posted Date: December 23rd, 2019

DOI: https://doi.org/10.21203/rs.2.19534/v1

License: (1) This work is licensed under a Creative Commons Attribution 4.0 International License. Read Full License 


\section{Abstract}

Background: Tackling neonatal infections and antibiotic resistance remains a challenge in low-income countries where neonatal mortality is high and antibiotic resistance is growing. The aim of this study was to identify bacterial etiologies and their drug resistance patterns in neonates admitted with diagnosis of sepsis to neonatal intensive care unit at Jimma Medical Center in Ethiopia.

Methods : A prospective longitudinal study was conducted from April to October 2018. A total of 313 clinical specimens (211 blood and 102 cerebrospinal fluid) were processed for 238 neonates suspected to have sepsis. Blood culture was done using BD BACTEC FX40 automated system. Bacterial identification and antibiotic susceptibility testing were done according to standard microbiological techniques.

Results : Bacterial etiologies were isolated from 62.1\% (131/211) and 3.9\% (4/102) of blood and cerebrospinal fluid cultures respectively. The predominant bacteria isolated from blood were Coagulase negative Staphylococcus $27.5 \%$ (36/131), followed by S. aureus 20.6\% (27/131), Klebsiella species 14.5\% (19/131), and Acinetobacter species $10.7 \%$ (14/131). Nearly $85 \%$ of the isolates were multidrug resistant, predominantly observed in Gramnegative bacteria. Among locally available antibiotics, only meropenem for Gram-negatives and vancomycin for Gram-positives were found to be largely effective.

Conclusion : Bacterial pathogens identified in the study were highly resistant to available antibiotics. Thus, an effort to reduce neonatal mortality in the setting should focus on improving diagnostics for neonatal infection and containment of antimicrobial resistance.

\section{Background}

Neonatal infection remains one of the most common causes of morbidity and mortality in newborns with over one million deaths annually; $99 \%$ of these deaths occur in resource-limited countries [1,2]. Neonatal sepsis can be classified based on the time of occurrence as early-onset and late-onset sepsis depending on the age at onset. Early-onset neonatal sepsis (EONS) is defined by its occurrence with seven days of life and is commonly caused by organisms acquired from the mother's genital tract during delivery. The most common causative organisms are Group B Streptococcus, Escherichia coli and Klebsiella pneumoniae. Late-onset neonatal sepsis (LONS) occurs after 7 days of life and is usually caused by pathogens acquired during hospitalization or delivery. The most common causative organisms are coagulase-negative Staphylococcus (CoNS), Staphylococcus aureus, Enterococcus species, and Enterobacteriaceae [1, 3, 4].

It is well documented that neonates with sepsis have an increased risk of developing meningitis, which on its own would deteriorates the prognosis of such neonates $[5,6]$. Data from both high- and low-income settings have shown that meningitis affects 0.3 to $3 \%$ of neonates with early onset neonatal sepsis $[7,8]$ and up to $30 \%$ of cases with LONS [6, 9-11]. Hence, there should be high index of suspicion for bacterial meningitis whenever neonatal sepsis is diagnosed, especially in cases of LONS $[9,10]$.

Early diagnosis and treatment of neonatal sepsis are essential to prevent severe and life-threatening complications. However, accurate diagnosis of neonatal infection remains a challenge, particularly in resource-limited settings, due to variable and non-specific clinical features and the difficulty of obtaining infection markers in the early stage [12]. Poor diagnostic facilities and lack of skilled health workforce in such settings add further challenge to achieve the goal of reducing neonatal sepsis globally. In real-life clinical practice, treatment is additionally challenging due to 
the absence of susceptibility patterns of bacterial etiologies of sepsis and the lack of accurate diagnostic markers $[13,14]$. Antibiotic treatment is also increasingly complicated by limited options and the emergence of high level and more extensive antimicrobial resistance against the existing antibiotics in several developing countries [15].

Tackling neonatal sepsis and antibiotic resistance is extremely challenging in low-income countries where neonatal mortality is high and antibiotic resistance is growing [16]. In these countries, isolation of etiologies of sepsis as well as determining drug susceptibility patterns of the organisms is difficult. As a result, overutilization of antibiotics is inevitable $[17,18]$. Consequently, multidrug resistant (MDR) organisms have emerged as important causes of neonatal sepsis in these settings in recent years. These extremely drug resistant pathogens include extendedspectrum beta-lactamase (ESBL)-producing Enterobacteriaceae [19-21], methicillin-resistant S.aureus (MRSA) [21, 22], and MDR Acinetobacter baumannii [23], among other MDR organisms. Different studies have shown that a high prevalence of sepsis due to MDR bacterial pathogens in intensive care settings dramatically increases mortality of newborns, especially in developing countries [2, 20, 24].

Essential data on the burden of severe bacterial infections in neonates and bacterial causes are scarce in Ethiopia, and the magnitude of antibiotic resistance remains unclear. Thus, a better understanding of the driving forces of bacterial sepsis and transmission is needed to help the fight against neonatal sepsis and antibiotic resistance. Hence, this study aimed to determine the profile and drug resistance patterns of bacteria causing sepsis in neonates admitted to a tertiary teaching hospital in Ethiopia.

\section{Materials And Methods}

\section{Setting}

Jimma Medical Center (JMC) is a university hospital in southwest Ethiopia with over 700 beds and catchment population of over 20 million. The study was conducted at the neonatal intensive care unit (NICU) of the hospital, which is a second level NICUs with a total bed capacity of 50 and an annual admission of 1800 to 2400 neonates. Neonates with a broad diversity of cases including infectious diseases, metabolic and genetic disorders, congenital malformations and surgical conditions are admitted and treated at the NICU. Neonates admitted to the NICU arrive from delivery units of the hospital itself and from various health facilities in the catchment area.

\section{Study design}

A prospective longitudinal study was conducted from April to October 2018. All neonates admitted to NICU with clinical diagnosis of sepsis and investigated with blood and/or CSF culture during the study period were included in the study.

\section{Data collection}

A structured case reporting format was used to collect background data and clinical information. One sample of 1-

3 milliliters venous blood was collected from the neonates by staff nurses. Additionally, 2-3ml of cerebrospinal fluid (CSF), in the absence of contraindications, was also collected by resident physician from neonates suspected to have sepsis. The specimens were collected following the principles of aseptic specimen collection and were immediately transported to the microbiology laboratory of JMC for processing and analysis.

\section{Isolation and identification of pathogens}


The blood specimens were inoculated into a BD BACTEC Peds Plus/F bottle. The bottles were then incubated in the BD BACTEC ${ }^{\text {TM }}$ FX40 automated culture machine for a maximum of five days until they were flagged as "negative" or "positive" for growth. Positive bottles were taken out from the machine and sub-cultured on Blood, Chocolate, and MacConkey agar (Oxoid, England). The CSF specimens, on the other hand, were directly inoculated on Blood, Chocolate and MacConkey agar (Oxoid, England) plates within 30 minutes of collection.

The chocolate and Blood agar plates from both blood and CSF specimens were incubated in a candle jar to create a condition of $5-10 \% \mathrm{CO}_{2}$. All the plates were incubated at $35-37^{\circ} \mathrm{C}$ aerobically for $18-24$ hours. After overnight incubation, all the inoculated plates were inspected and organisms grown on the plates were identified according to the standard microbiological identification techniques [25].

\section{Antimicrobial susceptibility testing}

Antibiotic susceptibility testing was carried out using Kirby-Bauer disc diffusion technique on Mueller-Hinton agar (Oxoid, England) according to Clinical Laboratory Standard Institute guidelines. Penicillin $(1 \mu \mathrm{g})$, oxacillin $(1 \mu \mathrm{g})$, cefoxitin $(30 \mu \mathrm{g})$, ampicillin $(10 \mathrm{mg})$, erythromycin $(15 \mathrm{mg})$, clindamycin $(2 \mathrm{mg})$, trimethoprim-sulphamethoxazole $(1.25 \mu \mathrm{g})$, amoxicillin/clavulanic acid $(10 \mathrm{mg})$, ceftazidime $(30 \mu \mathrm{g})$, cefepime $(30 \mu \mathrm{g})$, ceftriaxone $(30 \mu \mathrm{g})$, gentamicin (10mg), ciprofloxacin (5mg), tetracycline (30mg), chloramphenicol (30mg), ampicillin-sulbactam (10mg), vancomycin $(30 \mu \mathrm{g})$, and meropenem $(10 \mathrm{mg})$ were used. All the antibiotic discs used for the study were obtained from Oxoid Ltd., Basingstoke, Hampshire, UK. Reference strains of E. coli ATCC 25922 and S. aureus ATCC 25923 were used to control the reliability of antimicrobial susceptibility tests.

\section{Statistical analysis}

The data were entered using Epi Data version 3.1 and exported to SPSS version 25 and Microsoft excel for analysis. Descriptive statistics were used to show the frequency of bacterial agents and their drug resistance pattern.

\section{Ethical considerations}

Ethical approval was obtained from both Jimma University Institute of Health Institutional Review Board, Ethiopia (protocol number: IHRPGD/274/2018) and the Ethics Committee at the Medical Faculty of Ludwig Maximilian University of Munich, Germany. Written informed consent was obtained from the families or guardians of each neonate before they were recruited into the study. All the information collected were kept confidential and recorded in an anonymized fashion. All the microbiologic results of each neonate were immediately reported to the treating physician so that s/he could modify the treatment of the patient accordingly.

\section{Results}

Culture and antimicrobial susceptibility testing was performed for 313 clinical specimens (211 blood and 102 CSF) from 238 neonates suspected to have sepsis. Of these, 75 neonates had both blood and CSF cultures done. Overall, $63.5 \%$ (134/211) of the blood cultures and 3.9\% (4/102) of the CSF cultures were found to be positive for microbial growth. From these clinical specimens (blood and CSF), a total of 135 bacterial and 8 fungal etiologies were isolated. Sepsis episodes caused by Gram-positive bacteria, Gram-negative bacteria, and Candida species were 50.3\% (70/139), 43.9\% (61/139), and 5.8\% (8/139), respectively. Among the 131 bacterial pathogens isolated from blood culture, the predominant isolates were CoNS 27.5\% (36/131), S. aureus 20.6\% (27/131), Klebsiella spp. 
14.5\% (19/131), and Acinetobacter species 10.7\% (14/131) (Figure 1). Of these, double bacterial isolates were detected in cultures from five neonates.

From CSF samples, two Acinetobacterspp. and two K. pneumoniae were isolated. In one neonate, three types of bacterial pathogens were isolated simultaneously; $S$. aureus and Acinetobacter species from blood culture and $K$. pneumoniae from CSF.

CoNS - coagulase-negative Staphylococcus, GBS - group b Streptococcus, spp - species

Figure 1: Etiologies identified from blood culture in neonates admitted with sepsis to neonatal intensive care unit of Jimma Medical Center, Ethiopia

\section{Antibiotic susceptibility pattern of isolates}

The most common Gram-negative bacterial pathogens isolated in this study (from both blood and CSF) were Klebsiella spp., Acinetobacter spp., and Citrobacter spp. accounting for 32.3\% (21/65), 24.6\% (16/65), and 15.4\% $(10 / 65)$ of the isolates, respectively. All of the isolated Klebsiella spp. were resistant to ampicillin, amoxicillin/clavulanic-acid, cefoxitin, ceftriaxone, cefepime, chloramphenicol, sulfamethoxazole-trimethoprim, and tetracycline. Similarly, all isolates of Acinetobacterspp. were found to be resistant to ampicillin, amoxicillin/clavulanic acid, cefoxitin, ceftazidime, ceftriaxone, chloramphenicol, tetracycline and sulfamethoxazole-trimethoprim. However, all isolates of Klebsiella spp. were susceptible to meropenem, whereas $25 \%$ of Acinetobacter spp. were meropenem resistant. The other commonly isolated Gram-negative bacteria causing neonatal sepsis, Citrobacter spp., also showed MDR patterns, being resistant to ampicillin, amoxicillin/clavulanic acid, cefoxitin, ceftazidime, ceftriaxone, cefepime, and tetracycline. Three (30\%) of the isolated Citrobacter spp. were resistant to meropenem (Table 1).

Table 1: Antibiotic resistance patterns of Gram-negative bacterial pathogens in neonates admitted with sepsis to neonatal intensive care unit of Jimma Medical Center, Ethiopia 


\begin{tabular}{|c|c|c|c|c|c|c|c|}
\hline Antibiotics & $\begin{array}{l}\text { Klebsiella } \\
\text { spp. } \\
(n=21)\end{array}$ & $\begin{array}{l}\text { Acinetobacter } \\
\text { spp. }(n=16)\end{array}$ & $\begin{array}{l}\text { Citrobacter } \\
\text { spp. } \\
(n=10)\end{array}$ & $\begin{array}{l}\text { E. } \\
\text { coli } \\
(n=5)\end{array}$ & $\begin{array}{l}P . \\
\text { aeruginosa } \\
(n=5)\end{array}$ & $\begin{array}{l}\text { *Other } \\
\text { GNB } \\
(n=8)\end{array}$ & $\begin{array}{l}\text { Total } \\
(n=65)\end{array}$ \\
\hline Ampicillin & $100 \%$ & $100 \%$ & $100 \%$ & $100 \%$ & $100 \%$ & $100 \%$ & $100 \%$ \\
\hline Amoxicillin/clavulanate & $100 \%$ & $100 \%$ & $100 \%$ & $100 \%$ & $100 \%$ & $100 \%$ & $100 \%$ \\
\hline Cefoxitin & $100 \%$ & $100 \%$ & $100 \%$ & $60 \%$ & $100 \%$ & $100 \%$ & $100 \%$ \\
\hline Ceftazidime & $95 \%$ & $100 \%$ & $100 \%$ & $60 \%$ & $100 \%$ & $100 \%$ & $95 \%$ \\
\hline Ceftriaxone & $100 \%$ & $100 \%$ & $100 \%$ & $40 \%$ & $100 \%$ & $100 \%$ & $95 \%$ \\
\hline Cefepime & $100 \%$ & $81 \%$ & $100 \%$ & $40 \%$ & $100 \%$ & $100 \%$ & $91 \%$ \\
\hline Tetracycline & $100 \%$ & $100 \%$ & $100 \%$ & $80 \%$ & $100 \%$ & $100 \%$ & $98 \%$ \\
\hline Chloramphenicol & $100 \%$ & $100 \%$ & $90 \%$ & $20 \%$ & $0 \%$ & $88 \%$ & $74 \%$ \\
\hline $\begin{array}{l}\text { Trimethoprim- } \\
\text { sulphamethoxazole }\end{array}$ & $100 \%$ & $100 \%$ & $90 \%$ & $60 \%$ & $80 \%$ & $88 \%$ & $92 \%$ \\
\hline Ciprofloxacin & $76 \%$ & $31 \%$ & $30 \%$ & $20 \%$ & $20 \%$ & $63 \%$ & $48 \%$ \\
\hline Gentamycin & $76 \%$ & $50 \%$ & $80 \%$ & $40 \%$ & $20 \%$ & $63 \%$ & $62 \%$ \\
\hline Meropenem & $0 \%$ & $25 \%$ & $30 \%$ & $0 \%$ & $60 \%$ & $12.5 \%$ & $17 \%$ \\
\hline
\end{tabular}

* Includes: Enterobacter spp. (3), Providencia spp. (3), Serratia spp. (1), and Proteus mirabilis (1)

GNB - Gram-negative bacteria

Coagulase negative Staphylococci $(51.4 \% ; 36 / 70)$ and S. aureus (38.6\%; 27/70) were the most common Grampositive bacteria isolated from the study participants. The CoNS group showed high level of resistance to penicillin (89.0\%) and oxacillin (83.3\%). Similarly, S. aureus, showed a high rate of resistance against sulfamethoxazoletrimethoprim (88.9\%), penicillin (85.2\%), tetracycline (74.1\%) and erythromycin (70.4\%). Moreover, $63.0 \%$ of $S$. aureus were methicillin resistant (MRSA). On the other hand, the Micrococcus spp. identified in three neonates were found to be susceptible to almost all antibiotics tested (Table 2).

Table 2: Antibiotic resistance pattern of isolated Gram-positive bacterial pathogens in neonates admitted with sepsis to neonatal intensive care unit of Jimma Medical Center, Ethiopia 
Antibiotics

\begin{tabular}{lllll}
\hline Penicillin & $89 \%$ & $85 \%$ & $71.4 \%$ & $86 \%$ \\
\hline Oxacillin & $83 \%$ & $63 \%$ & $71.4 \%$ & $74 \%$ \\
\hline Cefoxitin & $83 \%$ & $63 \%$ & $71.4 \%$ & $74 \%$ \\
\hline Erythromycin & $56 \%$ & $70 \%$ & $57.1 \%$ & $61 \%$ \\
\hline Clindamycin & $33 \%$ & $33 \%$ & $57.1 \%$ & $36 \%$ \\
\hline Ceftriaxone & $78 \%$ & $67 \%$ & $57.1 \%$ & $73 \%$ \\
\hline Tetracycline & $81 \%$ & $74 \%$ & $71.4 \%$ & $76 \%$ \\
\hline Trimethoprim-sulphamethoxazole & $89 \%$ & $89 \%$ & $57.1 \%$ & $86 \%$ \\
\hline Vancomycin & $6 \%$ & $11 \%$ & $0 \%$ & $7 \%$
\end{tabular}

* Includes: Group B Streptococcus ( $\mathrm{n}=3)$, Micrococcus spp. $(\mathrm{n}=3)$, and L. monocytogenes $(\mathrm{n}=1)$

GPB - Gram-positive bacteria

Resistance to more than one agent in three or more antimicrobial categories was seen in $84.4 \%(114 / 135)$ of the bacterial isolates. MDR patterns were observed predominantly in Gram-negative bacterial pathogens. All of the isolated Klebsiella spp., Acinetobacter spp., P. aeruginosa, Providencia spp., Serratia spp., P. mirabilis, and $90 \%$ of Citrobacter spp. were MDR (Figure 2). Amongst the isolated Gram-negative MDR strains, one strain of each of Providencia spp., Klebsiella spp. and Citrobacterspp., and two strains of Acinetobacterspp. were resistant to all of the tested antibiotics, including carbapenem antibiotics.

MDR - multidrug resistant, NMDR - non-multidrug resistant, GNB - Gram-negative bacteria, GPB - Gram-positive bacteria.

Figure 2: Distribution of MDR and non-MDR patterns of bacterial isolates in neonates admitted with sepsis to neonatal intensive care unit of Jimma Medical Center, Ethiopia

Twenty-four, $10.1 \%(24 / 238)$ of the neonates died in the hospital. Of these, $66.7 \%(16 / 24)$ had positive blood and/or CSF culture and a total of 17 pathogens were identified from them. The predominant isolates in these groups were Klebsiella spp. $(\mathrm{n}=5)$, Acinetobacterspp. $(\mathrm{n}=2)$, P. aeruginosa $(\mathrm{n}=2)$ and Citrobacter species $(\mathrm{n}=2)$, all of which were MDR. E. coli and Enterobacter spp. isolated in this group were also found to be MDR. Though the sample size of this group is too small to determine any statistical association, it is highly possible that MDR organisms might have contributed to neonatal mortality at the hospital. It is also important to note here that $81 \%$ $(13 / 16)$ of the deaths in culture-confirmed cases occurred in patients identified with Gram-negative bacterial sepsis.

\section{Discussion}

In this study, blood culture was able to identify etiologic agents in $63.5 \%$ of neonates suspected to have sepsis. This proportion is higher than that in findings from recent similar studies in different parts of Ethiopia; $46.6 \%$ in Gondar [26], 44.7\% in Addis Ababa [27], and 29.4\% in Asella [28]. Our finding is also higher than in similar studies 
from other low- and middle-income countries; 49.7\% in Tanzania [14], 43.4\% in India [29], 16.9\% in Nepal [30], and $12.2 \%$ in Iran [31].

The high rate of culture positivity in our study may be explained by various reasons. First of all, the participants were neonates admitted to NICU only, excluding neonates in relatively stable medical conditions admitted to regular pediatric ward. Secondly, we used a highly sensitive automated blood culture system (BD BACTEC ${ }^{\text {TM }}$ Blood Culture) [32] unlike in most of the other studies mentioned above where manual systems were used. Thirdly, the fact that we used only one blood specimen for culture, contamination might have also contributed to increased culture positivity. Nevertheless, in such low-income setting where studies on neonatal infections are limited, such a finding may also be reflecting a reality on the ground, since sample taking was part of routine clinical procedures and not investigator initiated. However, involved staffs do undergo regular trainings on appropriate and sterile sampletaking techniques.

Group B Streptococcus, E. coli and K. pneumoniae in EONS and CoNS, S. aureus, and Enterococcus spp. in LONS are major cause of neonatal sepsis [1,3]. In our study however, the common etiologies identified were CoNS, S. aureus, Acinetobacter spp., Klebsiella spp. and Citrobacter spp. These bacteria are mostly acquired from the hospital environments, health professionals, and medical devices [33,34]. Moreover, as majority of our study participants $(84.1 \%)$ had early onset neonatal sepsis, the bacterial etiologies identified in our study somehow deviate from expectations $[1,3,4]$. One of the possible explanations is that about $68 \%$ of the neonates were delivered at the same hospital where high rate of hospital acquired infection by these organisms has been documented [35-37]. On the other hand, recent similar studies in Ethiopia have revealed that CoNS, S. aureus and Klebsiella spp. are major cause of neonatal sepsis in the country [26-28]. These evidences suggest that in settings where infection prevention and control practices are not properly implemented [38, 39]; infections by these agents constitute a significant threat to neonates and other vulnerable populations.

Concerning to drug resistance, the isolates in the present study were resistant against commonly used antibiotics in the area and high frequency of MDR bacterial pathogens were observed predominantly among the Gram-negative bacteria. Recent studies in Ethiopia, India, Nepal, and China also showed high levels of resistance in Gram-negative organisms against ampicillin (85\%, 78\%, 100\%, 80\%), respectively, and ceftriaxone (57\%, 100\%, 100\%, 50\%), respectively $[26,29,30,40]$. Low level of resistance was observed against meropenem (17\%) in Gram-negative bacteria and vancomycin (7\%) in Gram-positive bacteria. A potential explanation is that these two antibiotics are expensive and not widely available in the setting studied and hence less frequently prescribed. Among the older antibiotics, only ciprofloxacin (48\% resistance) and clindamycin (35\% resistance) showed better in vitro efficacy against Gram-negative and Gram-positive bacteria respectively.

The MDR patterns in both Gram-negative and Gram-positive bacteria in our study $(84.4 \%)$ is by far higher than national and global reports: Ethiopia (65\%) [26], Jordan (69\%) [23], and China (50\%) [40]. Moreover, the high rates of MRSA and Acinetobacter spp. resistant to almost all antibiotics available in the setting are serious concerns for the healthcare system in the region and beyond. Unavailability of microbiologic diagnostic facilities, poor infection prevention and control practices, lack of antimicrobial quality control, lack of control on antimicrobial utilization and absence of proper antimicrobial resistance surveillance system in the country and at the hospital in particular might have contributed to this alarming rate of MDR pattern.

The proportion of culture confirmed meningitis in neonates with sepsis is only 4\%. As most of the neonates had EONS, the finding is concordant with existing global evidences $[7,8]$. However, this finding cannot be generalized to 
all neonates admitted to the hospital and other settings in Ethiopia as our study has excluded neonates admitted to regular pediatric wards.

Our study is one of few studies on etiologies and AMR patterns in neonatal sepsis in Ethiopia. We believe that the findings in this study complement what has already been done and could provide important data for policy-level intervention to tackle neonatal mortality and antimicrobial resistance. However, the fact that our study is limited to one facility, a tertiary teaching hospital, and that only neonates admitted to NICU were included may limit the generalizability of the findings. Moreover, only one blood sample was taken from the neonates for culture, contamination during specimen collection might have contributed to the high rate of culture positivity in our study.

\section{Conclusions}

This study has shown that MDR CoNS, S. aureus, and Gram-negative bacilli (Klebsiella, Acinetobacter, and Citrobacter spp.) were the leading causes of neonatal sepsis at the hospital. The emergence of MDR pathogens in the country, including resistance to reserve antimicrobials and the unmet target in reduction of neonatal mortality, deserve intervention at policy-level. This should be done through development of locally acceptable and applicable guidelines, adhering to standard evidenced based practices, commitment to rational use of antimicrobials, improving diagnostic facilities and implementing, nationwide AMR surveillance system. Future research should also be carried out focusing on identification of appropriate local empiric therapies with improved susceptibility profiles for timely and effective treatment.

\section{Abbreviations}

AMR

antimicrobial resistance

ATCC

American Type Culture Collection

CoNS

Coagulase negative Staphylococcus

CSF

Cerebrospinal fluid

EONS

Early onset neonatal sepsis

ESBL

Extended spectrum beta lactamase

JMC

Jimma Medical Center

LONS

Late onset neonatal sepsis

MDR

Multidrug resistance

MRSA

Methicillin resistance Staphylococcus aureus

NICU

Neonatal intensive care unit 
Spp

Species

SPSS

Statistical Package for social science

\section{Declarations}

\section{Ethics approval and consent to participate}

This study was approved by the Institutional Review Board (IRB) of Jimma University Institute of Health ethical committee, Ethiopia and The Ethics Committee at the Medical Faculty of LMU Munich, Germany. Written informed consent was also obtained from parents and/or guardians of the neonate.

\section{Consent for publication}

Not applicable.

\section{Availability of data and materials}

The dataset will be available from the corresponding author on reasonable request.

\section{Competing interests}

All the authors declare that they have no competing interests regarding this work.

\section{Funding}

The study was funded by the Center for International Health $(\mathrm{CIH})$, German Academic Exchange Service (DAAD) through its Exceed program and the German Ministry of Economic Cooperation and Development. The funder had no any role in the design of the study and collection, analysis, and interpretation of data and in developing the manuscript.

\section{Authors' contributions}

EKG, SA, MB, MG, GKB, AW and GF conceptualized and designed the study protocol. BE, MG, MB, EKG, SA, NW and GT coordinated and supervised data collection. MG and EKG drafted the manuscript and all the authors critically reviewed and approved the final manuscript in its current form and agreed to be accountable for all aspects of the work.

\section{Acknowledgements}

This study was funded by Center for International Health $(\mathrm{CIH})$, German Academic Exchange Service (DAAD) through its Exceed program and the German Ministry of Economic Cooperation and Development. We thank all the data collectors (Nurses, Laboratory Personnel, and Residents) who are working in Jimma Medical Center, Jimma, Ethiopia.

\section{Authors' information}

${ }^{1}$ School of Medical Laboratory Science, Jimma University, Ethiopia 
${ }^{2}$ Department of Pediatrics and Child Health, Jimma University, Ethiopia

${ }^{3}$ Division for Infection Control and Environmental Health, Norwegian Institute of Public Health, Oslo, Norway

${ }^{4}$ Innlandet Hospital Trust, Division Gjøvik-Lillehammer, Gjøvik, Norway

${ }^{5}$ Division of Infectious Disease and Tropical Medicine, University Hospital (LMU), Munich

${ }^{6}$ Max von Pettenkofer-Institute (Medical Microbiology), LMU

${ }^{7}$ Department of Internal Medicine, Jimma University, Jimma, Ethiopia

${ }^{8}$ St.Paul's Hospital Millennium Medical College, Addis Ababa, Ethiopia

\section{References}

1. Seale AC, Mwaniki M, Newton CR, Berkley JA. Maternal and early onset neonatal bacterial sepsis: burden and strategies for prevention in sub-Saharan Africa. The Lancet Infectious Diseases. 2009;9(7):428-38.

2. Waters D, Jawad I, Ahmad A, Lukšić I, Nair H, Zgaga L, et al. Aetiology of community-acquired neonatal sepsis in low and middle income countries. Journal of global health. 2011;1(2):154.

3. Li Z, Xiao Z, Li Z, Zhong Q, Zhang Y, Xu F. 116 cases of neonatal early-onset or late-onset sepsis: A single center retrospective analysis on pathogenic bacteria species distribution and antimicrobial susceptibility. International journal of clinical and experimental medicine. 2013;6(8):693.

4. Bizzarro MJ, Raskind C, Baltimore RS, Gallagher PG. Seventy-five years of neonatal sepsis at Yale: 1928-2003. Pediatrics. 2005;116(3):595-602.

5. Aleem S, Greenberg RG. When to Include a Lumbar Puncture in the Evaluation for Neonatal Sepsis. Neoreviews. 2019;20(3):e124-e34.

6. Bhagat R, Hussain SQ, Gattoo IA, Wani SA. Incidence of meningitis in late onset sepsis. Int J Contemp Pediatr. 2015;2(2):7.

7. Simonsen KA, Anderson-Berry AL, Delair SF, Davies HD. Early-onset neonatal sepsis. Clin Microbiol Rev. 2014;27(1):21-47.

8. Polin RA, Committee on F, Newborn. Management of neonates with suspected or proven early-onset bacterial sepsis. Pediatrics. 2012;129(5):1006-15.

9. Kaul V, Harish R, Ganjoo S, Mahajan B, Raina SK, Koul D. Importance of obtaining lumbar puncture in neonates with late onset septicemia a hospital based observational study from north-west India. J Clin Neonatol. 2013;2(2):83-7.

10. Malbon K, Mohan R, Nicholl R. Should a neonate with possible late onset infection always have a lumbar puncture? Arch Dis Child. 2006;91(1):75-6.

11. Laving AM, Musoke RN, Wasunna AO, Revathi G. Neonatal bacterial meningitis at the newborn unit of Kenyatta National Hospital. East Afr Med J. 2003;80(9):7.

12. Hedegaard SS, Wisborg K, Hvas A-M. Diagnostic utility of biomarkers for neonatal sepsis-a systematic review. Infectious diseases. 2015;47(3):117-24. 
13. Sorsa A. Diagnostic Significance of White Blood Cell Count and C-Reactive Protein in Neonatal Sepsis; Asella Referral Hospital, South East Ethiopia. Open Microbiol J. 2018;12:209-17.

14. Kayange N, Kamugisha E, Mwizamholya DL, Jeremiah S, Mshana SE. Predictors of positive blood culture and deaths among neonates with suspected neonatal sepsis in a tertiary hospital, Mwanza- Tanzania. BMC pediatrics. 2010;10(1):39.

15. Folgori L, Ellis SJ, Bielicki JA, Heath PT, Sharland M, Balasegaram M. Tackling antimicrobial resistance in neonatal sepsis. The Lancet Global Health. 2017;5(11):e1066-e8.

16. Seale AC, Obiero CW, Berkley JA. Rational development of guidelines for management of neonatal sepsis in developing countries. Current opinion in infectious diseases. 2015;28(3):225.

17. Liu L, Oza S, Hogan D, Perin J, Rudan I, Lawn JE, et al. Global, regional, and national causes of child mortality in 2000-13, with projections to inform post-2015 priorities: an updated systematic analysis. The Lancet. 2015;385(9966):430-40.

18. Organization WH. Global antimicrobial resistance surveillance system ( GLASS) report: early implementation 2017-2018. 2018.

19. Biedenbach DJ, Moet GJ, Jones RN. Occurrence and antimicrobial resistance pattern comparisons among bloodstream infection isolates from the SENTRY Antimicrobial Surveillance Program (1997-2002). Diagnostic microbiology and infectious disease. 2004;50(1):59-69.

20. Zakariya BP, Bhat V, Harish BN, Babu TA, Joseph NM. Neonatal sepsis in a tertiary care hospital in South India: bacteriological profile and antibiotic sensitivity pattern. The Indian Journal of Pediatrics. 2011;78(4):413-7.

21. Chelliah A, Thyagarajan R, Katragadda R, Leela K, Babu RN. Isolation of MRSA, ESBL and AmpC- $\beta$-lactamases from neonatal sepsis at a tertiary care hospital. Journal of clinical and diagnostic research: JCDR. 2014;8(6):DC24.

22. Sharma P, Kaur P, Aggarwal A. Staphylococcus aureus-the predominant pathogen in the neonatal ICU of a tertiary care hospital in Amritsar, India. Journal of clinical and diagnostic research: JCDR. 2013;7(1):66.

23. Yusef D, Shalakhti T, Awad S, Khasawneh W. Clinical characteristics and epidemiology of sepsis in the neonatal intensive care unit in the era of multi-drug resistant organisms: a retrospective review. Pediatrics \& Neonatology. 2018;59(1):35-41.

24. Getabelew A, Aman M, Fantaye E, Yeheyis T. Prevalence of neonatal sepsis and associated factors among neonates in neonatal intensive care unit at selected governmental hospitals in Shashemene Town, Oromia Regional State, Ethiopia, 2017. International journal of pediatrics. 2018;2018.

25. Cheesbrough M. District Laboratory Practice in Tropical Countries. Cambridge: Cambridge University Press; 2005.

26. T GE, Moges F, Eshetie S, Yeshitela B, Abate E. Bacterial etiologic agents causing neonatal sepsis and associated risk factors in Gondar, Northwest Ethiopia. BMC Pediatr. 2017;17(1):137.

27. Shitaye D, Asrat D, Woldeamanuel Y, Worku B. Risk factors and etiology of neonatal sepsis in Tikur Anbessa University Hospital, Ethiopia. Ethiop Med J. 2010;48(1):11-21.

28. Sorsa A, Früh J, Stötter L, Abdissa S. Blood culture result profile and antimicrobial resistance pattern: a report from neonatal intensive care unit (NICU), Asella teaching and referral hospital, Asella, south East Ethiopia. Antimicrobial Resistance \& Infection Control. 2019;8(1):42.

29. Investigators of the Delhi Neonatal Infection Study c. Characterisation and antimicrobial resistance of sepsis pathogens in neonates born in tertiary care centres in Delhi, India: a cohort study. Lancet Glob Health. 
2016;4(10):e752-60.

30. Yadav NS, Sharma S, Chaudhary DK, Panthi P, Pokhrel P, Shrestha A, et al. Bacteriological profile of neonatal sepsis and antibiotic susceptibility pattern of isolates admitted at Kanti Children's Hospital, Kathmandu, Nepal. BMC Research Notes. 2018;11(1):301.

31. Mahallei M, Rezaee MA, Mehramuz B, Beheshtirooy S, Abdinia B. Clinical symptoms, laboratory, and microbial patterns of suspected neonatal sepsis cases in a children's referral hospital in northwestern Iran. Medicine. 2018;97(25):e10630-e.

32. Ahmad A, Iram S, Hussain S, Yusuf NW. Diagnosis of paediatric sepsis by automated blood culture system and conventional blood culture. J Pak Med Assoc. 2017;67(2):192-5.

33. Thapa S, Sapkota LB. Changing Trend of Neonatal Septicemia and Antibiotic Susceptibility Pattern of Isolates in Nepal. Int J Pediatr. 2019;2019:3784529.

34. Shete VB, Ghadage DP, Muley VA, Bhore AV. Acinetobacter septicemia in neonates admitted to intensive care units. J Lab Physicians. 2009;1(2):73-6.

35. Ali S, Birhane M, Bekele S, Kibru G, Teshager L, Yilma Y, et al. Healthcare associated infection and its risk factors among patients admitted to a tertiary hospital in Ethiopia: longitudinal study. Antimicrob Resist Infect Control. 2018;7:2.

36. Eshetu B, Gashaw M, Berhane M, Abdissa A, McClure EM, Goldenberg RL, et al. Intravenous fluid contaminated with Klebsiella oxytoca as a source of sepsis in a preterm newborn: Case report. American journal of infection control. 2019.

37. Gashaw M, Berhane M, Bekele S, Kibru G, Teshager L, Yilma Y, et al. Emergence of high drug resistant bacterial isolates from patients with health care associated infections at Jimma University medical center: a cross sectional study. Antimicrob Resist Infect Control. 2018;7:138.

38. Sahiledengle B, Gebresilassie A, Getahun T, Hiko D. Infection Prevention Practices and Associated Factors among Healthcare Workers in Governmental Healthcare Facilities in Addis Ababa. Ethiop J Health Sci. 2018;28(2):177-86.

39. Geberemariyam BS, Donka GM, Wordofa B. Assessment of knowledge and practices of healthcare workers towards infection prevention and associated factors in healthcare facilities of West Arsi District, Southeast Ethiopia: a facility-based cross-sectional study. Arch Public Health. 2018;76:69.

40. Li J-y, Chen S-q, Yan Y-y, Hu Y-y, Wei J, Wu Q-p, et al. Identification and antimicrobial resistance of pathogens in neonatal septicemia in China-A meta-analysis. International Journal of Infectious Diseases. 2018;71:89-93.

\section{Figures}




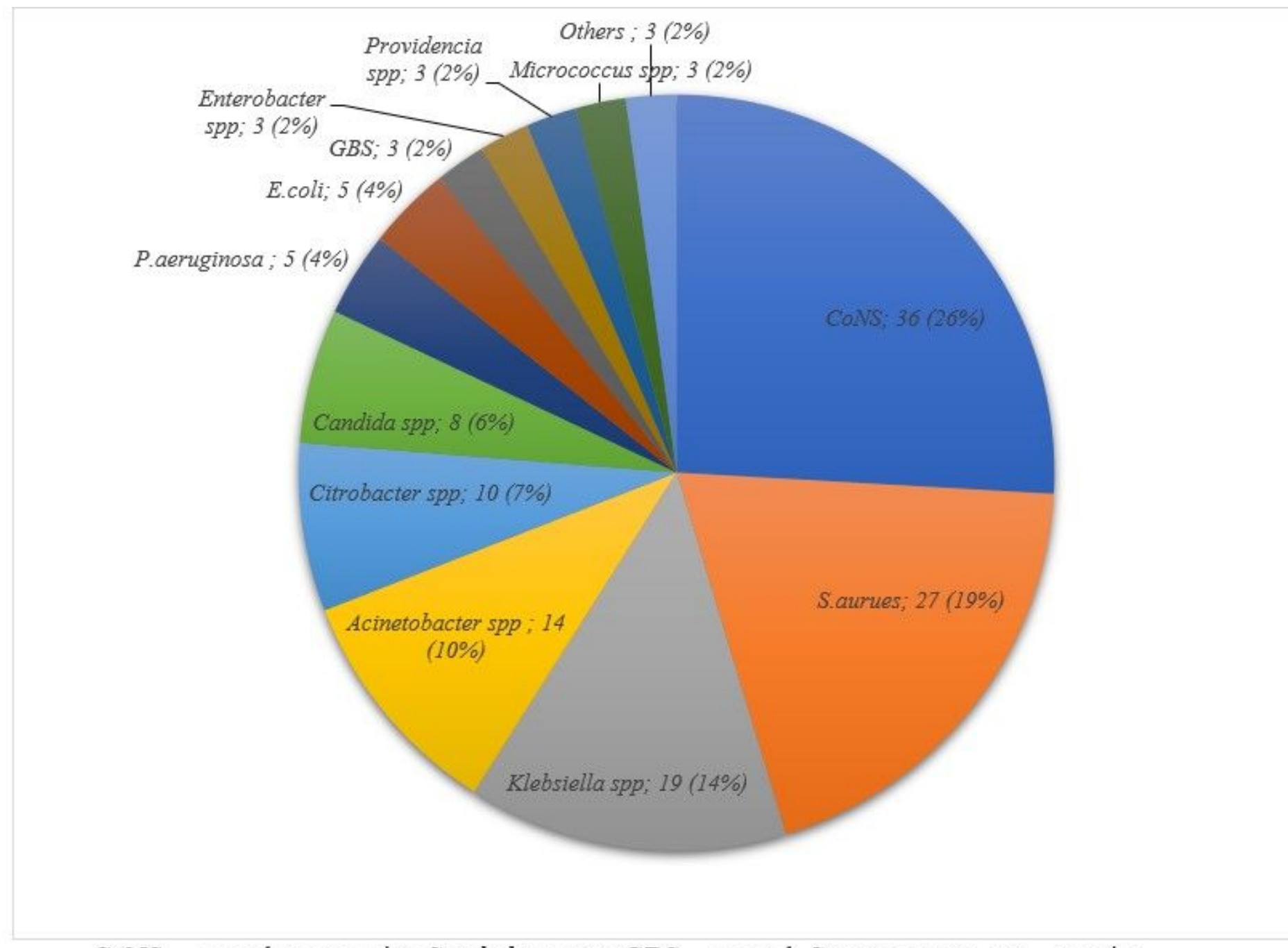

CoNS - coagulase-negative Staphylococcus, GBS - group b Streptococcus, spp - species

\section{Figure 1}

Etiologies identified from blood culture in neonates admitted with sepsis to neonatal intensive care unit of Jimma Medical Center, Ethiopia 


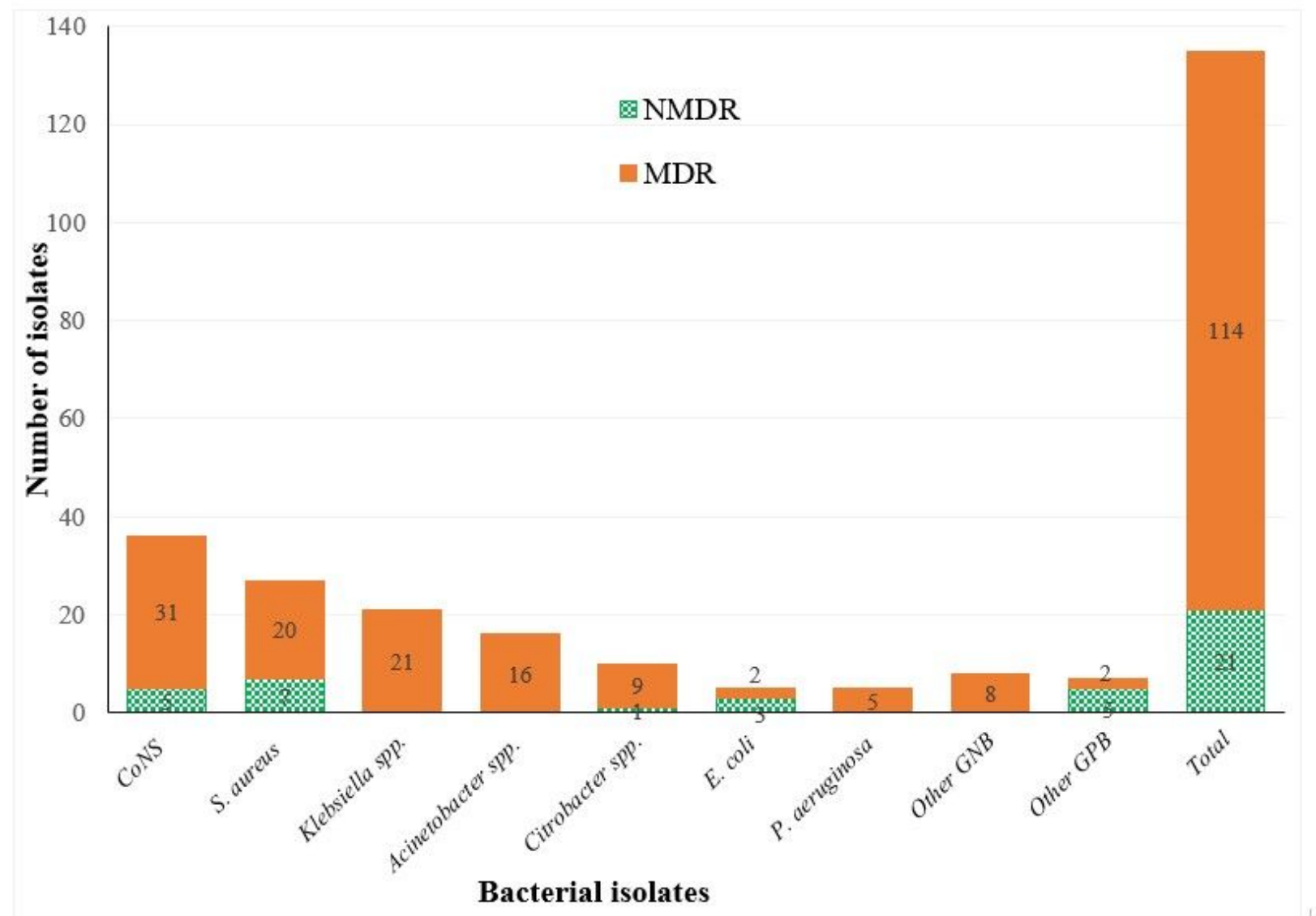

MDR - multidrug resistant, NMDR - non-multidrug resistant, GNB - Gram-negative bacteria, GPB - Grampositive bacteria.

Figure 2

Distribution of MDR and non-MDR patterns of bacterial isolates in neonates admitted with sepsis to neonatal intensive care unit of Jimma Medical Center, Ethiopia 